\title{
The Ablation of Thyroid Nodule's Afferent Arteries Before Radiofrequency Ablation: Preliminary Data
}

\author{
Chiara Offi ${ }^{1}$, Sara Garberoglio ${ }^{2}$, Giovanni Antonelli ${ }^{1}$, Maria Grazia Esposito ${ }^{1}$, \\ Umberto Brancaccio ${ }^{1}$, Claudia Misso ${ }^{1}$, Edoardo D'Ambrosio ${ }^{1}$, Daniela Pace ${ }^{3}$ \\ and Stefano Spiezia ${ }^{1 *}$

\begin{abstract}
1 Department of Endocrine and Ultrasound-guided Surgery, "Ospedale del Mare", Naples, Italy, ${ }^{2}$ Division of Endocrinology, Diabetology and Metabolism, Department of Medical Sciences, University of Turin, Turin, Italy, ${ }^{3}$ Department of Endocrinology, Valmontone Hospital, Valmontone, Italy
\end{abstract}

Induced radiofrequency thermal ablation is the cytoreductive treatment of symptomatic benign thyroid nodules, metastatic and recurrent thyroid tumors and papillary thyroid microcarcinomas. It is a safe and effective alternative to surgery and it allows to obtain satisfactory results in terms of volumetric reduction of the nodule with significant improvement in the quality of life. The trans-isthmic approach and the moving shot

OPEN ACCESS

Edited by:

Terry Francis Davies, Icahn School of Medicine at Mount Sinai, United States

Reviewed by: Giampaolo Papi, Local Health Unit of Modena, Italy Francesco Frasca,

University of Catania, Italy

*Correspondence: Stefano Spiezia stefanospiezia@tiroide.org

Specialty section: This article was submitted to Thyroid Endocrinology,

a section of the journa Frontiers in Endocrinology

Received: 23 May 2020 Accepted: 18 December 2020 Published: 11 February 2021

Citation:

Offi C, Garberoglio S, Antonelli G, Esposito MG, Brancaccio U, Misso C, D'Ambrosio E, Pace D and Spiezia S (2021) The Ablation of Thyroid Nodule's Afferent Arteries Before Radiofrequency Ablation: Preliminary Data.

Front. Endocrinol. 11:565000. doi: 10.3389/fendo.2020.565000 technique are the two basic techniques; however, an advanced technique, artery-first feeding radiofrequency ablation, has been developed and validated. We have prospectively included 29 consecutive patients who have undergone radiofrequency ablation (Group A) or artery- first vRFA (Group B). All included patients had a diagnosis of benign nodular goiter and they underwent a single session of radiofrequency ablation. All patients followed a follow-up program at 1 month, 3 months, and 6 months. Continuous variables (age, TSH value, basal volume of nodule, used Joule, time in second of the procedure, nodules' volume at 1-, 3-, and 6- months of follow-up and percentage of volume reduction at 1-, 3-, and 6- months of followup) were described as mean, standard deviation and range, while categorical variables (gender, nodule structure and nodule vascularization) were described as number of cases and percentage. Independent samples t-test were performed to compare the continuous variables. A Test of Proportions was applied to the categorical variables. The Fisher's exact test was used to analyze the gender. Statistical significance was considered in case of $p$-value $<0.05$. Solid structure and spongiform structure showed statistic differences with $p$-values of 0.022 and 0.023 respectively between two groups. The percentage of reduction at 1 month did not show a significant difference between two groups; instead, the percentage of volume reduction was decreased mostly in the Group B at 3 months and 6 months of follow-up with a p-value of 0.003 and 0.013 , respectively. The Joules/energy used showed a statistically significant difference ( $p$-value $=0.05$ ), more energy must be used in vascular radiofrequency ablation. These data allow us to hypothesize that vRFA may improve the effectiveness of the procedure, allowing for a reduction in volume more quickly. They were preliminary but promising results, clearly a larger series of cases and prolonged follow-up are needed to clarify and confirm our observations.

Keywords: radiofrequency ablation, thyroid nodules, technique, ultrasound, thermal ablation 


\section{INTRODUCTION}

Induced radiofrequency thermal ablation (RFA) is a minimally invasive technique developed to treat the symptoms and aesthetic outcomes of benign thyroid nodules. Thyroid nodules are the most common pathology of the thyroid gland, in most cases they are asymptomatic, stable over time and incidental; in a low percentage of cases, however, they have rapid growth associated with compression symptoms and unsightly outcomes (1). Until the last decade, these pathologies, when symptomatic, could only be treated with surgery. Recently, with the progress of minimally invasive techniques, thyroid nodules can be treated with various minimally invasive techniques including RFA (2).

RFA, performed by expert hands can be considered a safe and effective alternative to surgery for the treatment of selected pathologies. The RFA technique allows to obtain satisfactory results in terms of volumetric reduction of the nodule with a significant improvement in the quality of life. Patients treated with RFA ablation refer reduction of compression symptoms and discomfort, previously related to the presence of a mass in the neck. The trans-isthmic approach and the moving shot technique are the two basic techniques validated in many studies; however, an advanced technique, vascular radiofrequency ablation (vRFA), has been developed and validated (3).

Various International guidelines, indeed, consider RFA treatment a valid alternative to surgery in the case of symptomatic benign thyroid nodularities. Moreover, RFA may be an indication in the treatment of microcarcinoma, in recurrent thyroid cancers, in lymph node metastases and in cases where surgery is contraindicated or rejected by the patient (2-9). Especially in the treatment of papillary thyroid carcinoma recent data suggest the use of RFA as an alternative treatment to surgery $(3,4,10-12)$.

The aim of this study is to evaluate the percentage of nodular volume reduction at 1 month, 3 months, and 6 months in patients treated with RFA and in patients treated with vRFA. The goal is to evaluate the difference between the two techniques in the time of reduction of the nodular volume and to identify the superiority of one technique compared to the other.

\section{MATERIALS AND METHODS}

We have prospectively collected data of 29 consecutive patients who underwent RFA or artery- first vRFA at Department of Endocrine and Ultrasound-guided Surgery of "Ospedale del Mare", Naples, Italy. All patients had a diagnosis of nodular goiter and underwent radiofrequency ablation of thyroid nodule between January 2018 and August 2019.

We included patients aged over 18 years and not pregnant with: single thyroid nodule of the thyroid lobe with benign

Abbreviations: CD, Color Doppler; FNC, fine needle cytology; PD, Power Doppler; RFA, radiofrequency ablation; SD, standard deviation; TgAb, thyroglobulin antibody; TPOAb, Thyroperossidase antibody; TSH, thyrostimulating hormone; US, ultrasound; vRFA, vascular radiofrequency ablation. cytological diagnosis (Thy2/TIR2 or Bethesda II) to two consecutive fine needle cytology (FNC) $(13,14)$; complete medical history, complete preoperative blood tests and complete follow-up. We excluded patients with: presence of multiple thyroid nodule in a thyroid lobe; cystic nodules or predominantly cystic nodules on ultrasound (cystic portion $>50 \%$ of the nodule); cytological diagnosis of TIR1c, TIR3a, TIR3b, TIR4 and TIR5; non diagnostic cytological report (TIR1) to one FNC; autoimmune thyroid disease; TSH $<0.15 \mathrm{mU} / \mathrm{L}$ or $\mathrm{TSH}>3.5 \mathrm{mU} / \mathrm{L}$; previous thyroid radiofrequency ablation or ethanol injection; neck surgery within 5 years; previous radiation therapy; previous cancers. The flow chart (Figure 1) shows the patients enrolled according to the inclusion and exclusion criteria.

We divided the population in two groups based on RFA technique used. Group A included RFA patients. Group B included, on the other hand, patients subjected to vRFA. All patients underwent coagulation index and blood cells count sample, thyroid function samples, thyroid antibody samples, twice FNC, thyroid and later cervical lymph nodes B-mode US examination and fiberoptic-laryngoscopy tests in the 30 days prior to ultrasonographic ablation techniques. Clinical and demographic data (age, gender, baseline nodule volume, nodule structure, nodule vascularization, nodule function and a complete anamnesis) were obtained at first examination and were collected in an electronic database.

The US examination was conducted with a 7.5-12 MHz linear probe or with a convex probe in very large nodules equipped with $\mathrm{CD}$ and PD nodules (MyLab ${ }^{\mathrm{TM}}$ ClassC and $\mathrm{MyLab}^{\mathrm{TM}} 9$ Platform, Esaote Biomedica, Genova Italy) and assessing the volume of the nodule, the structure and the vascularization. The

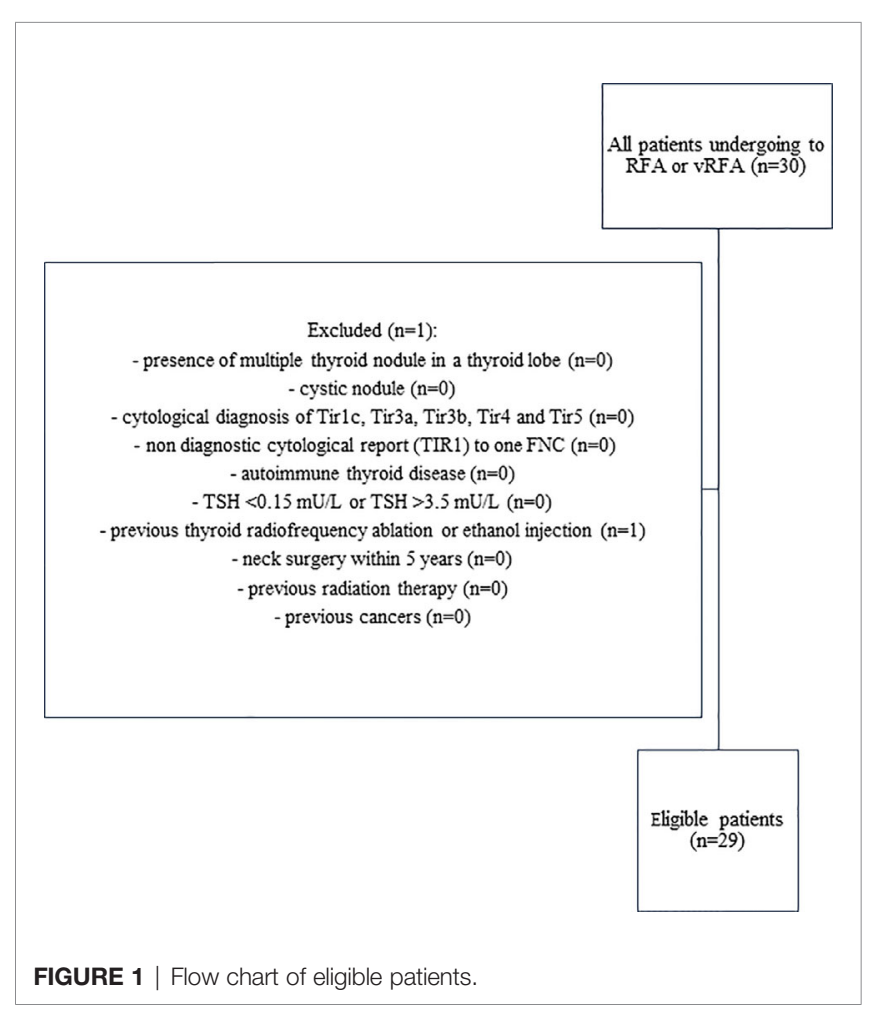


basal volume of the nodule was automatically calculated with ellipsoid equation by the software with the measurements of depth, width and length in longitudinal and transverse scans. The structure was defined as solid (fluid component was less than $10 \%$ of volume), spongiform (cystic areas occupied less than $50 \%$ of the nodule's volume) and predominantly solid (solid component was more than $50 \%$ of the volume). The vascularity was studied by CD examination and classified as peri-nodular, intra-nodular and both peri- and intra- nodular. Cytology findings, according to SIAPEC-IAP classification, were collected and we included patients with twice TIR2 at two consecutive FNCs.

The examined variables, relating to the technique, were the Joules (energy used to deliver the power of one watt for one second) used and the time expressed in seconds. Radiofrequency ablation was performed with the M-3004 generator and the RFTTIP 0710N, $7 \mathrm{~cm}$ length, $1 \mathrm{~cm}$ exposed tip (RF Medical Co., South Korea) inserted with US guidance.

The patients were submitted to a single RFA session, in supine position, in neck hyperextension and with a skin and pericapsular anesthesia. The skin local anesthesia was done in the needle insertion site in the midline. During the treatment, all patients had a feeling of compression at the neck's level, which did not require the interruption of the procedure. All patients were discharged $3 \mathrm{~h}$ after the procedure, after ultrasound examination. No cases of major or minor complications were observed.

The trans-isthmic approach and the moving shot technique are performed under ultrasound (US) guidance. The transisthmic approach allows the treatment of the left and right thyroid nodules by inserting the RFA electrode in the neck midline to lateral direction. It shows advantages over other approaches. Before reaching the target, the electrode crosses much of the thyroid parenchyma limiting involuntary excursions of the needle-electrode due to patients' involuntary movements (speaking, swallowing or coughing). It prevents the leakage of hot material from the thyroid parenchyma during the procedure. This approach also allows continuous ultrasound monitoring to minimize the risk of complications or side effects due to thermal injuries in surrounding structures (recurrent laryngeal nerve, carotid artery, internal jugular vein, trachea and esophagus) (5-7, 15-17). Moreover, the use of Color Doppler (CD) and/or Power Doppler (PD) prevents serious bleeding, identifying the blood vessels along the path of the electrode.

A radiofrequency generator (M-3004, RF Medical Co., Ltd., South Korea), connected to the needle-electrode, produces an alternating electric current (between 200 and 1,200 kHz) which causes ions movement in adjacent tissues of the tip. The ions vibrate rapidly producing energy that is transformed in heat, raising local temperature between $60^{\circ} \mathrm{C}$ and $100^{\circ} \mathrm{C}$. The high temperature induces a protein denaturation of cell membrane and the irreversible death of the cells of the tissues around the tip. The energy decreases exponentially in the length of the needle and the remote tissues are heated due to thermal conduction. This needle protection mechanism prevents tissues away from the target from undergoing coagulation necrosis due to the heat generated (18). At the beginning of the procedure, the temperature necroses all around the tip. US transient hyperechoic area is displayed and to the touch a vibration is perceived, described as a "shot".

The RFA has benefited from the moving shot technique as the thyroid nodules mainly have an ellipsoidal appearance and it is difficult to treat them with the fixed technique. The periphery of the thyroid nodules is undertreated or overtreated with the fixed technique. Using the moving shot technique, instead, the nodule is ideally divided into multiple smaller units, each to be ablated separately. The subunits are smaller on the periphery and larger in the central area and in regions far from critical structures. The procedure begins with the treatment of the deeper and more peripheral areas, as the heat generated causes the appearance of transient hyperechoic air bubbles that reduce the acoustic window for ultrasound monitoring, moreover, saving the "dangerous triangle" (the nodular periphery area close to trachea) to avoid thermal injury to the recurrent laryngeal nerve. It continues with the treatment of superficial areas of the nodule. The moving shot technique allows to obtain an ellipsoidal ablation area, similar to the morphology of the frequent thyroid nodules. The treatment ends when the entire nodule appears completely replaced by transient hyperechoic areas $(3,5,10,19-22)$.

The anesthetic infiltration of skin and pericapsular tissue is performed before the RFA: the needle is inserted at the midline of the anterior neck to anesthetize the skin. Once the skin has infiltrated, the needle is pushed in lateral direction, depending on the right or left position of the nodule, up to the virtual space between the strap muscles and the thyroid capsule. A solution with lidocaine $2 \%$ is injected and appears as an anechoic band, which gradually disappears deeply into antero-superior mediastinum, and laterally tools the main vessels of the neck. The risk of major complications decreases with local anesthesia, because the operator can monitor the pain, an early indicator of heat propagation, while a moderate sedation or a general anesthesia prevent the patients' active collaboration and the early manifestation of complications such as changes in tone of voice, cough or pain $(10,15,23)$.

The thyroid upper polar nodules are usually vascularized by arterial branches of the upper thyroid artery that derives from the external carotid artery and mainly supplies the upper and anterior part of the thyroid gland. The nodules of the lower pole of the thyroid are vascularized by the lower thyroid artery, which derives from the thyrocervical trunk and provides the posteroinferior parts of the gland.

The vRFA is a technique used to treat the hypervascular thyroid nodule. The artery-first vRFA is used in hypervascularized nodules that show an evident feeding artery. Authors propose this technique to reduce the dispersion of the generated heat, in the nodules with important vascularization (24). The feeding artery is detected with a CD and PD study (Figure 2) and the use of artery-first vRFA decreases the complications and increases the techniques efficiency. Intranodular linear echogenicities appear in the target nodule when the feeding artery is ablated due to air microbubbles in the arterioles 


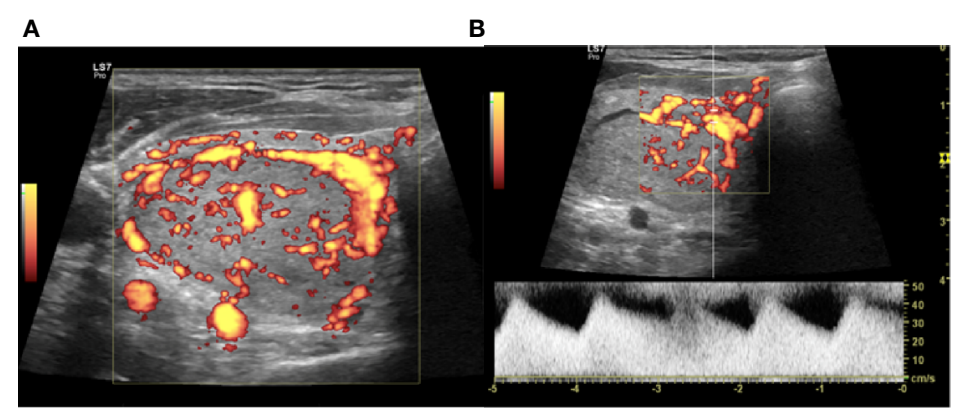

FIGURE 2 | Right lobe nodule, intraoperative transverse ultrasound (US) scan. (A) Power Doppler (PD) evaluation of the nodule to be treated with vascular radiofrequency ablation (VRFA). (B) Identification at PD and PWD of the main afferent artery.

(Figure 3). In a few cases, it's possible to have wedge-shaped hypoechoic area after the ablation due to the infarction of the area vascularized by the ablated feeding artery (25).

We carried out the thermo-ablative treatment of an afferent arterial branch of nodule as a first step of RFA treatment, improving the moving shot technique, in order to obtain a better ablation of nodular tissue, amplifying the volume of the induced necrosis (Figure 4) (3, 22, 25).

In the follow-up program we assessed the nodule volume and its percentage of reduction at 1 month, 3 months and 6 months. We assessed the volume with the ultrasound examination, evaluating the 3 diameters studied in the two projections. The reduction percentage was calculated comparing the follow-up volume with the basal volume.

Statistical analysis was performed with SPSS version 23 (SPSS $^{\odot}$, Chicago, IL, USA). Continuous variables were described as mean, standard deviation (SD) and range, while categorical variables were described as number of cases and percentage. The population was divided in patients who underwent RFA and patients who underwent vRFA. Independent samples t-test were performed to compare the continuous variables (Joule, procedure's time in second, basal volume, volume at 1 month, \% of volume reduction at 1 month, volume at 3 months, $\%$ of volume reduction at 3 months, volume at 6 months and \% of volume reduction at 6 months). A Test of Proportions was applied to the categorical variables (number of patients, nodule structure and vascularization). The Fisher's exact test was used to analyze the gender. Statistical significance was considered in case of p-value $<0.05$.

Written informed consent was obtained from all participants. All procedures were performed in accordance with the Helsinki Declaration.

\section{RESULTS}

Twenty-nine patients (10 males and 19 females) with a single benign thyroid nodule were enrolled in the study for the treatment with radiofrequency ablation. Patients were divided into two groups based on the treatment they were subjected to, Group A treated with RFA (12 cases) and Group B treated with vRFA (17 cases). Population's mean age was 55.03 years $( \pm 10.9$ SD) without a statistical difference between two groups. Patients included were euthyroid and they had normal values of Thyroglobulin Antibody (TgAb), Thyroperossidase Antibody (TPOAb) and calcitonin (Table 1). In a case of subclinical hyperthyroidism (TSH $0.12 \mathrm{mU} / \mathrm{L}$ ) thyroid scintigraphy was performed prior to treatment and it identified a non-

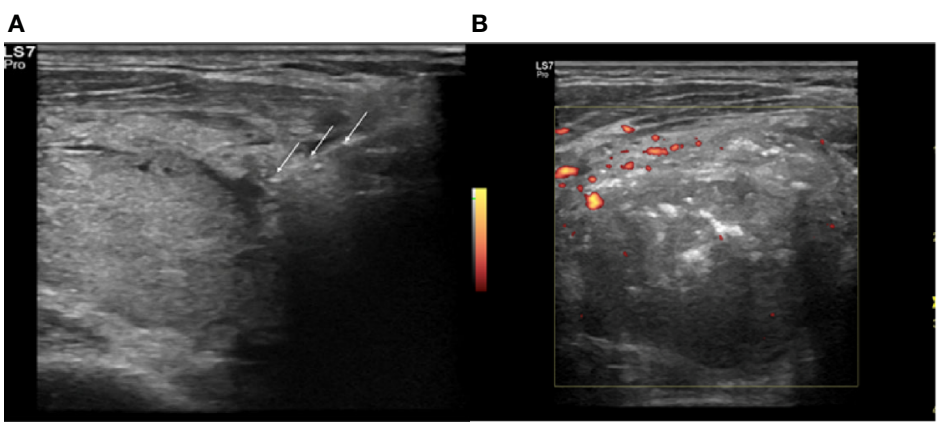

FIGURE 3 | Right lobe nodule, intraoperative transverse ultrasound (US) scan. (A) Insertion of the electrode (white arrows) in the identified arterial afferent pole. (B) Power Doppler (PD) evaluation at the end of the procedure, absence of peri- and intranodular vasculartization. 


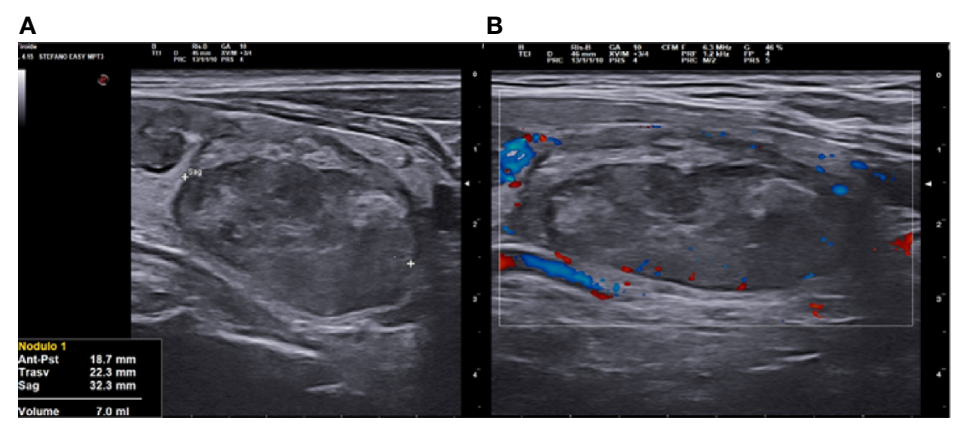

FIGURE 4 | Right lobe nodule, longitudinal ultrasound (US) scan at 3 months follow up after vascular radiofrequency ablation (vRFA). (A) The nodule shrunk from 18.5 to $7 \mathrm{ml}$ in 3 months, strongly hypoechoic, solid. (B) at Color Doppler (CD) evaluation, absence of peri- and intranodular vascular signal.

TABLE 1 | Baseline demographic and clinical data of the study population.

\begin{tabular}{|c|c|c|c|c|}
\hline Characteristics & Population & Group A & Group B & p-value \\
\hline Patients (\%) & $29(100)$ & $12(41.4)$ & $17(58.6)$ & 0.14 \\
\hline Mean age in years $\pm S D$ (range) & $55.03 \pm 10.90(28-80)$ & $57.50 \pm 7.28(46-69)$ & $53.29 \pm 12.80(28-80)$ & 0.315 \\
\hline \multicolumn{5}{|l|}{ Gender (\%) } \\
\hline Male & $10(34.5)$ & $4(13.8)$ & $6(20.7)$ & 0.99 \\
\hline Female & $19(65.5)$ & 8 (86.2) & $11(79.3)$ & \\
\hline Mean TSH in $m U / L \pm S D$ (range) & $1.03 \pm 0.49(0.12-2.21)$ & $0.93 \pm 0.42(0.54-1.78)$ & $1.11 \pm 0.54(0.12-2.21)$ & 0.337 \\
\hline Mean basal volumes $\pm S D$ (range) & $26.90 \pm 19.09(1.60-74)$ & $21.15 \pm 20.34(1.60-74)$ & $30.96 \pm 17.65(10.40-70.10)$ & 0.178 \\
\hline \multicolumn{5}{|l|}{ Nodule structure (\%) } \\
\hline Solid & $9(31)$ & $7(58)$ & $2(11.8)$ & 0.023 \\
\hline Spongiform & $18(62)$ & $4(33.5)$ & $14(82.4)$ & 0.022 \\
\hline Predominantly solid & $2(7)$ & $1(8.5)$ & $1(5.8)$ & 0.99 \\
\hline \multicolumn{5}{|l|}{ Nodule vascularization (\%) } \\
\hline Peri-nodular & 8 (27.6) & $5(41.7)$ & $3(17.7)$ & 0.315 \\
\hline Intra-nodular & $1(3.5)$ & $1(8.5)$ & $0(0)$ & 0.858 \\
\hline Intra-peri-nodular & $20(68.9)$ & $6(49.8)$ & $14(82.3)$ & 0.14 \\
\hline
\end{tabular}

SD, standard deviation; TSH, thyro-stimulating hormone.

functioning nodule. The mean Thyroid-stimulating hormone (TSH) value was $1.03 \mathrm{mU} / \mathrm{L}( \pm 0.49 \mathrm{mU} / \mathrm{L} \mathrm{SD})$ and showed no significant differences between the two populations (Table 1).

Ultrasound nodule structure was summarized in Table $\mathbf{1 .}$ Nodules with solid, spongiform and predominantly solid structure were selected. Solid structure and spongiform structure showed statistically significant differences with $\mathrm{p}$ values of 0.022 and 0.023 respectively. The solid structure nodules were treated more with RFA (seven cases with RFA versus two cases with vRFA), while the spongiform nodules were treated more with vRFA (four cases with RFA versus 14 cases with vRFA).

Baseline nodule vascularization characteristics are summarized in Table $\mathbf{1}$ and do not show significant difference.

At baseline, the nodule volume ranged 1.60 to $74 \mathrm{ml}$ with a mean of $26.90 \pm 19.09 \mathrm{ml}$ (Table 1). Considering the two groups separately, the Group A' mean volume was $21.15 \pm 20.34 \mathrm{ml}$, in the other hand, Group B' mean volume was $30.96 \pm 17.65 \mathrm{ml}$ with a $\mathrm{p}$-value $=0.178$. The variables of the ablative techniques are illustrated in Table 2. The Joules/energy used showed a statistically significant difference between the two techniques, with a mean of $29663.83 \pm 21040.14 \mathrm{~J} / \mathrm{s}$ in Group A and a mean of $48275.47 \pm 26029.28 \mathrm{~J} / \mathrm{s}$ (p-value=0.05), showing that more energy must be used in vRFA. The execution time expressed in seconds showed no significant difference. Both techniques were conducted by two skilled operators of a tertiary thyroid center.

After radiofrequency ablation treatment the mean volume of nodule decreased progressively (Table 3, Figure 5): it was $14.15 \pm$ $11.37 \mathrm{ml} 1$ month after treatment; $9.26 \pm 8.48 \mathrm{ml} 3$ months after treatment and $6.41 \pm 6.53 \mathrm{ml} 6$ months after treatment (p-value of $0.279,0.784$ and 0.451 , respectively). The nodule volume decreased gradually during 1 month of follow-up with a mean of $14.15 \mathrm{ml}$ versus $26.9 \mathrm{ml}$ at baseline. The percentage of reduction at 1 month was $47.26 \%$ in study population without a significant difference between Group A and Group B ( $p$-value= 0.369). Subsequently, percentage of volume reduction was decreased mostly in the Group B at 3 months and 6 months of follow-up with a p-value of 0.003 and 0.013 , respectively. At 3 months of follow-up, Group B showed a mean percentage reduction of $72.71 \pm 8.99 \%$ compared to the Group A's mean percentage reduction of $56.28 \pm 14.55 \%$. Moreover, at 6 months follow-up, Group B showed a mean percentage reduction of $83.01 \pm 5.04 \%$ 
TABLE 2 | Radiofrequency ablation characteristics (SD, standard deviation).

\begin{tabular}{|c|c|c|c|c|}
\hline Characteristics & Population & Group A & Group B & $\begin{array}{c}\mathrm{p}- \\
\text { value }\end{array}$ \\
\hline Mean used Joule $\pm S D$ (range) & $\begin{array}{c}40,574.10 \pm 25,457.69(4,560- \\
98,000)\end{array}$ & $\begin{array}{c}29,663.83 \pm 21,040.14(4,560- \\
71,180)\end{array}$ & $\begin{array}{c}48,275.47 \pm 26,029.28(9,800- \\
98,000)\end{array}$ & 0.05 \\
\hline $\begin{array}{l}\text { Mean time in seconds } \pm S D \\
\text { (range) }\end{array}$ & $638.55 \pm 299.33(192-1,320)$ & $537.58 \pm 244.47(192-985)$ & $709.82 \pm 320.42(204-1,320)$ & 0.129 \\
\hline
\end{tabular}

TABLE 3 | Thyroid nodule volume in follow-up (SD, standard deviation).

\begin{tabular}{|c|c|c|c|c|}
\hline Characteristics & Population & Group A & Group B & p-value \\
\hline 1 month volume $\pm S D$ (range) & $14.15 \pm 11.37(1.60-45)$ & $11.38 \pm 12.36(1.60-45)$ & $16.09 \pm 10.55(4.12-38.40)$ & 0.279 \\
\hline 1 month $\%$ volume variation $\pm S D$ (range) & $47.26 \pm 16.5(0-74.26)$ & $43.45 \pm 22.67(0-69.54)$ & $49.95 \pm 10.23(35.58-74.26)$ & 0.369 \\
\hline 3 months volume $\pm S D$ (range) & $9.26 \pm 8.48(0.95-38)$ & $9.78 \pm 10.8(0.95-38)$ & $8.88 \pm 6.73(1.60-25)$ & 0.784 \\
\hline 3 months $\%$ volume variation $\pm S D$ (range) & $65.91 \pm 14.04(36.64-85.88)$ & $56.28 \pm 14.55(36.64-76.93)$ & $72.71 \pm 8.99(57.14-85.88)$ & 0.003 \\
\hline 6 months volume $\pm S D$ (range) & $6.41 \pm 6.53(0.21-31)$ & $7.68 \pm 9.11(0.21-31)$ & $5.51 \pm 3.92(0.90-14.40)$ & 0.451 \\
\hline 6 months $\%$ volume variation $\pm S D$ (range) & $77.64 \pm 12.07(48.07-91.34)$ & $70.04 \pm 15.04(48.07-89.72)$ & $83.01 \pm 5.04(75.89-91.34)$ & 0.013 \\
\hline
\end{tabular}

compared to Group A's mean percentage reduction of $70.04 \pm$ $15.04 \%$. Data showed that the percentage of nodule reduction was not dissimilar between the two groups after one month from the ablative treatment. At 3 months, instead, there was a significant percentage of major reduction in patients treated with vRFA. This statistical significance remained unchanged at 6 months, registering a greater volume reduction percentage in patients treated with vRFA than in patients treated with RFA.

\section{DISCUSSION}

Numerous studies have shown that radiofrequency ablative treatment is an effective and safe treatment in symptomatic benign nodules of the thyroid $(1,12,16,19,21,26)$. International guidelines show that radiofrequency is effective in the treatment of microcarcinomas and recurrent carcinomas of less than $2 \mathrm{~cm}$ in diameter, not only in the treatment of symptomatic benign nodules $(2,10)$. Any study has shown the difference in the efficacy of RFA versus vRFA with artery-first step ablation techniques. The aim of our study was to evaluate the difference in the effectiveness of RFA and vRFA with a 6month follow-up in symptomatic benign thyroid nodule.

Effective cytoreduction occurs when large volumes of necrosis are rapidly obtained with ablative treatments (22). In our population study, the percentage of volume reduction was similar between the two groups at 1-month follow-up, but it's statistically different in Group B at 3- and 6-months follow-up. The percentage of reduction at 1 -month is not different in the two groups due to infarction of the nodule treated with vRFA. The infarction is due to a reduction in the vascularization of the nodule and to a reduction in venous drainage (3). This scenario changes at 3 and 6 months of follow-up, in which there is a reduction in inflammation due to necrosis and a resumption of venous drainage with the removal of the necrotic cells from the treated nodule. Some Authors suggest that a volumetric reduction of treated nodule is from 50 to $80 \%$ after 6 months and from 70 to $90 \%$ after two years. In our cases we had a volume reduction of $43.45 \%, 56.28 \%$, and $70.04 \%$ at 1-, 3-, and 6- months in Group A and a volume reduction of $49.95 \%, 72.71 \%$, and $83.01 \%$ at 1-, 3-, and 6-months in Group B. The percentage of volume reduction is in line with the Literature data with a
A

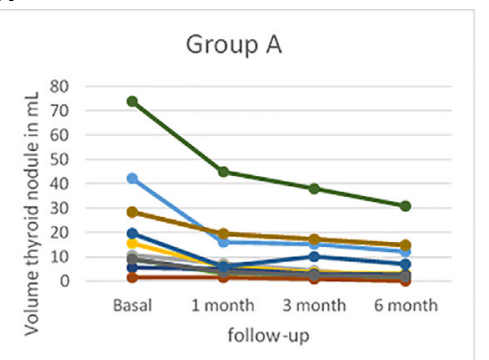

B

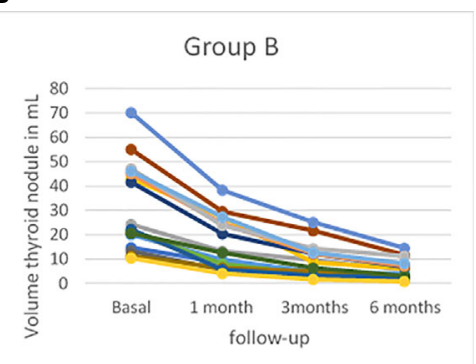

FIGURE 5 | Profiles of thyroid volume assessed by B-mode ultrasound (US) at baseline and at 1-, 3-, and 6-month follow-up (A) in patients treated with radiofrequency thermal ablation (RFA) (Group $A$ ) and $(\mathbf{B})$ in patients treated with vRFA (Group B). 
reduction of more than $50 \%$ of the volume already after 3 months of treatment in both techniques (21). In the future we will publish a study with a wider case history and a longer followup, to evaluate the percentage of reduction over the years and a possible regrowth of the nodule, comparing the two methods. The long-term efficacy of the treatment is assessed by observing the absence of nodular vital tissue regrowth, which can be demonstrated during ultrasound examinations carried out in the period following the treatment. Nodular regrowth, in the other hand, has been associated with the presence of inadequately treated nodular margins (21, 26-28).

In our population study, the nodules' feeding artery is ablated with RFA before starting the ablative treatment. The rational of RFA is to increase the local temperature in order to induce tissue necrosis. Many benign thyroid nodules are hyper-vascularized. The adjacent vascular structures can dissipate the heat generated during the procedure reducing the treatment's effectiveness. The early ablation of afferent artery reduces the vascularization of the nodule and the heat dissipation. Moreover, the afferent artery ablation reduces the risk of bleeding and of hematomas. The hemorrhage, during the RFA, leads to increase of volume and to obstacle the thermal conduction $(3,22)$. Our population shows a difference compared to the structure of the nodule. In Group A we have $58 \%$ of the population with a solid echostructure of the nodule, while in the Group B we have $82.4 \%$ with a spongiform echostructure of the nodule. The significant difference is affected by the choice of technique. vRFA can be used in the treatment of nodules with a feeding artery visible and nodules with a spongiform echostructure are nodules of greater size and intensely vascularized. Deandrea et al. suggest that spongiform nodule and cystic nodule have a greater reduction volume compared to solid nodules (21). Deandrea et al. hypothesize that in the nodules with a greater fluid component and in the more vascularized ones, the heat induced by the electrode produces more steam and the steam improves the thermocoagulation process (21). These data are in line with the ones observed in our study population. The energy utilized (J/s) shows significant difference. In vRFA group we have utilized increased energy ( 29663.83 vs $48275.47 \mathrm{~J} / \mathrm{s}$ ) as the method foresees two treatment steps: a first phase in which the nutritious artery is ablated and a second phase in which the nodule is ablated.

Park et al. describe an US hyperechoic linear streak at the nodule periphery after the afferent artery ablation. The US image is due to overheating which induces the appearance of microbubbles inside the arterioles. In some cases, on the other hand, it's possible to view a wedge-shaped hypoechoic area due to ischemia. The ischemic area is a rare finding especially in hypervascularized nodules due to the presence of several collateral circles (3).

Our preliminary experience, conducted on symptomatic thyroid nodules, shows that the radiofrequency treatment is a safe and an effective treatment. The method allows to reduce the compression symptoms, to obtain high percentages of reduction of the volume of the nodule even with just one treatment, to treat the nodule with the most effective technique based on the anatomical conditions and is squeezed to a risk of complications absent when performed by expert hands.
We achieved a significant percentage of greater volume reduction in the vRFA group compared to patients treated only with RFA. At follow-up, peripheral vascularization appears less evident on $\mathrm{CD}$, in patients undergoing vRFA than in those treated only with RFA. These considerations allow us to hypothesize that vRFA may improve the effectiveness of the procedure, allowing for an improvement in symptoms and a reduction in volume more quickly. They were preliminary but promising results, clearly a larger series of cases and prolonged follow-up are needed to clarify and confirm our observations.

\section{DATA AVAILABILITY STATEMENT}

The raw data supporting the conclusions of this article will be made available by the authors, without undue reservation.

\section{ETHICS STATEMENT}

The studies involving human participants were reviewed and approved by Ethics Committee Campania Center Prot. no 375/C.E $18-2020$ oss of 09/15/2020. The patients/participants provided their written informed consent to participate in this study.

\section{AUTHOR CONTRIBUTIONS}

All authors contributed significantly to the present research and reviewed the entire manuscript. CO participated substantially in conception, design and execution of the study and in the analysis and interpretation of the data; also participated substantially in the drafting and editing of the manuscript. SG participated substantially in conception, design and execution of the study and in the analysis and interpretation of the data; also participated substantially in the drafting and editing of the manuscript. GA participated substantially in conception and design of the manuscript and in the analysis and interpretation of the data. ME participated substantially in conception and design of the manuscript and in the analysis and interpretation of the data. UB participated substantially in conception and design of the manuscript and in the analysis and interpretation of the data. CM participated substantially in conception and design of the manuscript and in the analysis and interpretation of the data. ED'A participated substantially in conception and design of the manuscript and in the analysis and interpretation of the data. DP participated substantially in conception and design of the manuscript and in the analysis and interpretation of the data. SS participated substantially in conception, design and execution of the study and in the analysis and interpretation of the data; also participated substantially in the drafting and editing of the manuscript. All authors contributed to the article and approved the submitted version. 


\section{REFERENCES}

1. Papini E, Gugliemi R, Pacella CM. Laser, radiofrequency, and ethanol ablation for the management of thyroid nodules. Curr Opin Endocrinol Diabetes Obes (2016) 23(5):400-6. doi: 10.1097/MED.0000000000000282

2. Haugen BR, Alexander EK, Bible KC, Doherty GM, Mandel SJ, Nikiforov YE, et al. 2015 American Thyroid Association Management Guidelines for Adult Patients with Thyroid Nodules and Differentiated Thyroid Cancer: The American Thyroid Association Guidelines Task Force on Thyroid Nodules and Differentiated Thyroid Cancer. Thyroid (2016) 26(1):1-133. doi: 10.1089/ thy. 2015.0020

3. Park HS, Baek JH, Park AW, Chung SR, Choi YJ, Lee JH. Thyroid Radiofrequency Ablation: Updates on Innovative Devices and techniques. Korean J Radiol (2017) 18(4):615-23. doi: 10.3348/kjr.2017.18.4.615

4. Wu R, Luo Y, Tang J, Yang M, Li J, Zhang Y, et al. Ultrasound-guided radiofrequency ablation for papillary thyroid microcarcinoma: a retrospective analysis of 198 patients. Int J Hyperthermia (2020) 37(1):168-74. doi: 10.1080/02656736.2019.1708480

5. Lee J, Shin JH, Hahn SY, Park KW, Choi JS. Feasibility of Adjustable Electrodes for Radiofrequency Ablation of Benign Thyroid Nodules. Korean J Radiol (2010) 21(3):377-83. doi: 10.3348/kjr.2019.0724

6. Pacella CM, Bizzarri G, Spiezia S, Bianchini A, Guglielmi R, Crescenzi A, et al. Thyroid tissue: US-guided percutaneous laser thermal ablation. Radiology (2004) 232(1):272-80. doi: 10.1148/radiol.2321021368

7. Spiezia S, Garberoglio R, Di Somma C, Deandrea M, Basso E, Limone PP, et al. Efficacy and safety of radiofrequency thermal ablation in the treatment of thyroid nodules with pressure symptoms in elderly patients. J Am Geriatr Soc (2007) 55(9):1478-9. doi: 10.1111/j.1532-5415.2007.01306.x

8. Rosato L, Avenia N, Bernante P, De Palma M, Gulino G, Nasi PG, et al. Complications of thyroid surgery: analysis of a multicentric study on 14,934 patients operated on in Italy over 5 years. World J Surg (2004) 28(3):271-6. doi: 10.1007/s00268-003-6903-1

9. Kim JH, Baek JH, Sung JY, Min HS, Kim KW, Hah JH, et al. Radiofrequency ablation of low-risk small papillary thyroidcarcinoma: preliminary results for patients ineligibe for surgery. Int J Hyperthermia (2017) 33(2):212-9. doi: 10.1080/02656736.2016.1230893

10. Kim JH, Baek JH, Lim HK, Ahn HS, Baek SM, Choi JY, et al. 2017 Thyroid Radiofrequency Ablation Guideline: Korean Society of Thyroid Radiology. Korean J Radiol (2018) 19(4):632-55. doi: 10.3348/kjr.2018.19.4.632

11. Zhang M, Tufano RP, Russell JO, Zhang Y, Zhang Y, Qiao Z, et al. Ultrasound-Guided Radiofrequency Ablation Versus Surgery for Low-Risk Papillary Thyroid Microcarcinoma: Results of Over 5 Years' Follow-Up. Thyroid (2020) 30(3):408-17. doi: 10.1089/thy.2019.0147

12. Papini E, Pacella CM, Solbiati LA, Achille G, Barbaro D, Bernardi C, et al. Minimally-invasive treatments for benign thyroid nodules: a Delphi-based consensus statement from the Italian minimally-invasive treatments of the thyroid (MITT) group. Int J Hyperthermia (2019) 36(1):376-82. doi: 10.1080/ 02656736.2019.1575482

13. Nardi F, Basolo F, Crescenzi A, Fadda G, Frasoldati A, Orlandi F, et al. Italian consensus for the classification and reporting of thyroid cytology. J Endocrinol Invest (2014) 37:593-9. doi: 10.1007/s40618-014-0062-0

14. Cibas ES, Ali SZ. The Bethesda System for Reporting Thyroid Cytopathology. Thyroid (2009) 7(1):1159-65. doi: 10.1089/thy.2009.0274

15. Barile A, Quarchioni S, Bruno F, Ierardi AM, Arrigoni F, Giordano AV, et al. Interventional radiology of the thyroid gland: critical review and state of the art. Gland Surg (2018) 132-46. doi: 10.21037/gs.2017.11.17

16. Jung SL, Baek JH, Lee JH, Shong YK, Sung JY, Kim KS, et al. Efficacy and Safety of Radiofrequency Ablation for Benign Thyroid Nodules: A Prospective
Multicenter Study. Korean J Radiol (2018) 19(1):167-74. doi: 10.1530/EJE-180685

17. Lim HK, Cho SJ, Baek JH, Lee KD, Son CW, Son JM, et al. Guided Radiofrequency Ablation for Low-Risk Papillary Thyroid Microcarcinoma: Efficacy and Safety in a Large Population. Korean J Radiol (2019) 20 (12):1653-61. doi: 10.3348/kjr.2019.0192

18. Beak JH, Lee JH, Valcavi R, Pacella CM, Rhim H, Naet DG. Thermal ablation for benign thyroid nodules: radiofrequency and laser. Korean J Radiol (2011) 12(5):525-40. doi: 10.3348/kjr.2011.12.5.525

19. Hong J, Baek JH, Choi YJ, Lee JH, Lim HK, Shong YK, et al. Radiofrequency ablation is a thyroid function-preserving treatment for patients with bilateral benign thyroid nodules. J Vasc Interv Radiol (2015) 26(1):55-61. doi: 10.1016/ j.jvir.2014.09.015

20. Xiaoyin T, Ping L, Dan C, Min D, Jiachang C, Tao W, et al. Risk Assessment and Hydrodissection Technique for Radiofrequency Ablation of Thyroid Benign Nodules. J Cancer (2018) 9(17):3058-66. doi: $10.7150 /$ jca. 26060

21. Deandrea M, Trimboli P, Garino F, Mormile A, Magliona G, Ramunni MJ, et al. Long-Term Efficacy of a Single Session of RFA for Benign Thyroid Nodules: A Longitudinal 5-Year Observational Study. J Clin Endocrinol Metab (2019) 104(9):3751-6. doi: 10.1210/jc.2018-02808

22. Mauri G, Gennaro N, Lee MK, Baek JH. Laser and radiofrequency ablations for benign and malignant thyroid tumors. Int J Hyperthermia (2019) 36 (2):13-20. doi: 10.1080/02656736.2019.1622795

23. Piccioni F, Poli A, Templeton LC, Templeton W, Rispoli M, Vetrugno L, et al. Anesthesia for Percutaneous Radiofrequency Tumor Ablation (PRFA): A Review of Current Practice and Techniques. Local Reg Anesth (2019) 12:12737. doi: 10.2147/LRA.S185765

24. Pillai K, Akhter J, Chua TC, Shehata M, Issan Al-Alem NA, Morris DL. Heat sink effect on tumor ablation characteristics as observed in monopolar radiofrequency, bipolar radiofrequency, and microwave, using ex vivo calf liver model. (2015) 94(9):e580. doi: 10.1097/MD.0000000000000580

25. Ha EJ, Baek JH, Lee JH. Ultrasonography-Based Thyroidal and Perithyroidal Anatomy and Its Clinical Significance. Korean J Radiol (2015) 16(4):749-66. doi: 10.3348/kjr.2015.16.4.749

26. Feldkamp J, Grünwald F, Luster M, Lorenz K, Vorländer C, Führer D. NonSurgical and Non- Radioiodine Techniques for Ablation of Benign Thyroid Nodules: Consensus Statement and Recommendation. Exp Clin Endocrinol Diabetes (2020) 128(10):687-92. doi: 10.1055/a-1075-2025

27. Sim JS, Baek JH, Lee J, Cho W, Jung SI. Radiofrequency ablation of benign thyroid nodules: depicting early sign of regrowth by calculating vital volume. Int J Hyperthermia (2017) 33(8):905-10. doi: 10.1080/02656736. 2017.1309083

28. Vuong NL, Dinh LQ, Bang HT, Thuy TTM, Bac NH, Vy TT. Radiofrequency Ablation for Benign Thyroid Nodules: 1-Year Follow-Up in 184 Patients. World J Surg (2019) 43(10):2447-53. doi: 10.1007/s00268-019-05044-5

Conflict of Interest: The authors declare that the research was conducted in the absence of any commercial or financial relationships that could be construed as a potential conflict of interest.

Copyright (c) 2021 Offi, Garberoglio, Antonelli, Esposito, Brancaccio, Misso, D'Ambrosio, Pace and Spiezia. This is an open-access article distributed under the terms of the Creative Commons Attribution License (CC BY). The use, distribution or reproduction in other forums is permitted, provided the original author(s) and the copyright owner(s) are credited and that the original publication in this journal is cited, in accordance with accepted academic practice. No use, distribution or reproduction is permitted which does not comply with these terms. 